PROCEEDINGS OF THE

AMERICAN MATHEMATICAL SOCIETY

Volume 139, Number 2, February 2011, Pages 747-756

S 0002-9939(2010)10724-4

Article electronically published on September 24, 2010

\title{
PARAMETRIC BING AND KRASINKIEWICZ MAPS: REVISITED
}

\author{
VESKO VALOV \\ (Communicated by Alexander N. Dranishnikov)
}

\begin{abstract}
Let $M$ be a complete metric $A N R$-space such that for any metric compactum $K$ the function space $C(K, M)$ contains a dense set of Bing (resp., Krasinkiewicz) maps. It is shown that $M$ has the following property: If $f: X \rightarrow Y$ is a perfect surjection between metric spaces, then $C(X, M)$ with the source limitation topology contains a dense $G_{\delta}$-subset of maps $g$ such that all restrictions $g \mid f^{-1}(y), y \in Y$, are Bing (resp., Krasinkiewicz) maps. We apply the above result to establish some mapping theorems for extensional dimension.
\end{abstract}

\section{INTRODUCTION}

All spaces in the paper are assumed to be metrizable and all maps continuous. By $C(X, M)$ we denote all maps from $X$ into $M$. Unless stated otherwise, all function spaces are endowed with the source limitation topology.

In this paper we extend some results from [17] and 21] concerning parametric Bing and Krasinkiewicz maps. A space $M$ is said to be a Krasinkiewicz space [17] if for any compactum $X$ the function space $C(X, M)$ contains a dense subset of Krasinkiewicz maps. Here, a map $g: X \rightarrow M$, where $X$ is compact, is said to be Krasinkiewicz [14] if every continuum in $X$ is either contained in a fiber of $g$ or contains a component of a fiber of $g$. The class of Krasinkiewicz spaces contains all Euclidean manifolds and manifolds modeled on Menger or Nöbeling spaces, all polyhedra (not necessarily compact), as well as all cones with compact bases (see [10, 14, 15, 16], 17]).

Our result concerning parametric Krasinkiewicz maps is as follows:

Theorem 1.1. Let $M$ be a complete Krasinkiewicz ANR-space and $f: X \rightarrow Y a$ perfect surjection between metric spaces. Then $C(X, M)$ contains a dense $G_{\delta}$-set of maps $g$ such that all restrictions $g \mid f^{-1}(y), y \in Y$, are Krasinkiewicz maps.

Theorem 1.1 was established in [17] in the case that $Y$ is strongly countable dimensional or $M$ is a closed convex subset of a Banach space and $Y$ is a $C$-space.

The second part of the paper is devoted to Bing maps. Recall that a map $f$ between compact spaces is said to be a Bing map [11] provided all fibers of $f$

Received by the editors December 22, 2008 and, in revised form, January 6, 2009.

2010 Mathematics Subject Classification. Primary 54F15, 54F45; Secondary 54E40.

Key words and phrases. Bing maps, Krasinkiewicz maps, continua, metric spaces, absolute neighborhood retracts, extensional dimension.

The author was partially supported by NSERC Grant 261914-08.

(C)2010 American Mathematical Society Reverts to public domain 28 years from publication 
are Bing spaces. Here, a compactum is a Bing space if each of its subcontinua is indecomposable. Following Krasinkiewicz [9], we say that a space $M$ is a free space if for any compactum $X$ the function space $C(X, M)$ contains a dense subset consisting of Bing maps. The class of free spaces is quite large, it contains all $n$ dimensional manifolds $(n \geq 1)$ [9], the unit interval [11], all locally finite polyhedra [19], all manifolds modeled on the Menger cube $M_{2 n+1}^{n}$ or the Nöbeling space $N_{2 n+1}^{n}$ [19], as well as all 1-dimensional locally connected continua [19].

Our second result is the following theorem concerning parametric Bing maps.

Theorem 1.2. Let $f: X \rightarrow Y$ be a perfect surjection between metric spaces. Then, for every complete $A N R$ free space $M$ the function space $C(X, M)$ contains a dense $G_{\delta}$-set of maps $g$ such that all restrictions $g \mid f^{-1}(y), y \in Y$, are Bing maps.

Theorem 1.2 was established in 21] in the case that $Y$ is strongly countable dimensional or $M$ is a closed convex subset of a Banach space and $Y$ is a $C$-space.

Theorem 1.2 is applied in the last Section 4 to show that some mapping theorems for extensional dimension established in 14 in the realm of compact metric spaces remain valid for general metric spaces.

The function space $C(X, M)$ appearing in this paper is endowed with the source limitation topology whose neighborhood base at a given function $f \in C(X, M)$ consists of the sets $B_{\rho}(f, \epsilon)=\{g \in C(X, M): \rho(g, f)<\epsilon\}$, where $\rho$ is a fixed compatible metric on $M$ and $\epsilon: X \rightarrow(0,1]$ runs over continuous positive functions on $X$. The symbol $\rho(f, g)<\epsilon$ means that $\rho(f(x), g(x))<\epsilon(x)$ for all $x \in X$. It is well known that for metrizable spaces $X$ this topology does not depend on the metric $\rho$ and it has the Baire property provided $M$ is completely metrizable.

\section{PARAMETRIC KRASINKIEWICZ MAPS}

This section contains the proof of Theorem 1.1. We fix a complete Krasinkiewicz $A N R$-space $M$. Then, by [21, Lemma 2.1], $M$ admits a complete metric $\rho$ generating its topology and having the following extension property:

- If $P$ is a paracompact space, $A \subset P$ a closed set and $\phi: A \rightarrow M$ is a map, then for every continuous function $\alpha: P \rightarrow(0,1]$ and every map $h: A \rightarrow M$ with $\rho(h(z), \phi(z))<\alpha(z) / 8$ for all $z \in A$, there exists a map $\bar{h}: P \rightarrow M$ extending $h$ such that $\rho(\bar{h}(z), \phi(z))<\alpha(z)$ for all $z \in P$.

Suppose $f: X \rightarrow Y$ is a perfect surjective map between metric spaces and $d$ is a metric generating the topology of $X$. For every $A \subset X$ and $\delta>0$, let $B(A, \delta)=$ $\{x \in X: d(x, A)<\delta\}$. If $y \in Y$ and $m, n \geq 1$, then following the notation from [17, we denote by $\mathcal{K}_{f}(m, n, y)$ the set of all maps $g \in C(X, M)$ such that:

- For each subcontinuum $L \subset f^{-1}(y)$ with $\operatorname{diam} g(L) \geq 1 / n$ there exists $x \in L$ such that $C\left(x, g \mid f^{-1}(y)\right) \subset B(L, 1 / m)$. Here, $g \mid f^{-1}(y)$ is the restriction of $g$ over $f^{-1}(y)$ and $C\left(x, g \mid f^{-1}(y)\right)$ denotes the component of the fiber $g^{-1}(g(x)) \cap f^{-1}(y)$ of $g \mid f^{-1}(y)$ containing $x$.

For a closed set $H \subset Y$ let $\mathcal{K}_{f}(m, n, H)$ be the intersection of all $\mathcal{K}_{f}(m, n, y)$, $y \in H$. We also denote by $\mathcal{K}_{f}(H)$ the set of all maps $g \in C(X, M)$ such that $g \mid f^{-1}(y)$ is a Krasinkiewicz map for every $y \in H$. It was established in [17] that each $\mathcal{K}_{f}(m, n, H)$ is open in $C(X, M)$ and $\mathcal{K}_{f}(H)=\bigcap_{m, n \in \mathbb{N}} \mathcal{K}_{f}(m, n, H)$.

Below $\mathbb{I}^{k}$ is the $k$-dimensional cube and $\mathbb{S}^{k-1}$ its boundary. 
Lemma 2.1. Let $\pi: Z \rightarrow \mathbb{I}^{k}$, where $Z$ is a metric compactum and $k \geq 1$. Suppose $g_{0} \in C(Z, M)$ with $g_{0} \in \mathcal{K}_{\pi}\left(\mathbb{S}^{k-1}\right)$. Then for every $\epsilon>0$ there exists a map $g \in C(Z, M)$ such that $g \in \mathcal{K}_{\pi}\left(\mathbb{I}^{k}\right), g$ is $\epsilon$-homotopic to $g_{0}$ and $g \mid \pi^{-1}\left(\mathbb{S}^{k-1}\right)=$ $g_{0} \mid \pi^{-1}\left(\mathbb{S}^{k-1}\right)$.

Proof. Since $M$ is an $A N R$, any two sufficiently close maps from $X$ into $M$ are homotopic. Hence, we need to prove that for every $\epsilon>0$ there exists a map $g \in \mathcal{K}_{\pi}\left(\mathbb{I}^{k}\right)$ which is $\epsilon$-close to $g_{0}$ and $g\left|\Omega=g_{0}\right| \Omega$, where $\Omega=\pi^{-1}\left(\mathbb{S}^{k-1}\right)$. According to [17. Proposition 2.4], each set $T(m, n)=\mathcal{K}_{\pi}\left(m, n, \mathbb{I}^{k}\right)$ is open in $C(Z, M)$.

Claim 1. Let $g \in C(Z, M)$ with $g\left|\Omega=g_{0}\right| \Omega$. Then for any $m, n \geq 1$ and $\delta>0$ there exists $h \in T(m, n)$ such that $h\left|\Omega=g_{0}\right| \Omega$ and $h$ is $\delta$-close to $g$.

We fix $m, n \geq 1$. Since $g\left|\Omega=g_{0}\right| \Omega, g \in \mathcal{K}_{\pi}(m, n, y)$ for every $y \in \mathbb{S}^{k-1}$. Then, by 17. Lemma 2.3], there exist a neighborhood $V_{y}$ of $y$ in $\mathbb{I}^{k}$ and $\delta_{y}>0$ satisfying the following condition: For every $y^{\prime} \in V_{y}$ and $g^{\prime} \in C(Z, M)$ with $\rho\left(g^{\prime}(x), g(x)\right)<\delta_{y}$ for all $x \in \pi^{-1}\left(y^{\prime}\right)$ we have $g^{\prime} \in \mathcal{K}_{\pi}\left(m, n, y^{\prime}\right)$. Choose finitely many $y_{i} \in \mathbb{S}^{k-1}, i \leq s$, such that $V=\bigcup_{i \leq s} V_{y_{i}}$ covers $\mathbb{S}^{k-1}$. Let $F=\mathbb{I}^{k} \backslash V$ and $\eta=\min \left\{\delta, \delta_{y_{i}}: i \leq s\right\}$. Because $M$ is a Krasinkiewicz space, there is a map $g_{1} \in \mathcal{K}_{\pi}\left(\mathbb{I}^{k}\right)$ which is $(\eta / 8)$-close to $g$. Then the map $g_{2}: \Omega \cup \pi^{-1}(F) \rightarrow M, g_{2}|\Omega=g| \Omega$ and $g_{2}\left|\pi^{-1}(F)=g_{1}\right| \pi^{-1}(F)$, is $(\eta / 8)$-close to $g \mid \Omega \cup \pi^{-1}(F)$. According to the extension property of $(M, \rho), g_{2}$ can be extended to a map $h: Z \rightarrow M$ which is $\eta$-close to $g$. We have $h \in \mathcal{K}_{\pi}(m, n, y)$ for all $y \in \mathbb{I}^{k}$. Indeed, this follows from the choice of $V_{y_{i}}$ and $\delta_{i}, i \leq s$, when $y \in V$, and from the fact that $g_{1} \in \mathcal{K}_{\pi}\left(\mathbb{I}^{k}\right)$ when $y \in F$. Hence, $h \in T(m, n)$, which completes the proof of the claim.

Now, we arrange all $T(m, n), m, n \geq 1$, as a sequence $\left\{T_{j}: j \geq 1\right\}$. Using the above claim and the openness of each $T_{j}$ in $C(Z, M)$, we construct by induction a sequence of positive numbers $\left\{\epsilon_{j}\right\}_{j \geq 0}$ and a sequence of maps $\left\{g_{j}\right\}_{j \geq 1} \subset C(Z, M)$ satisfying the following conditions for any $j \geq 0$ :

- $\epsilon_{0}=\epsilon / 3$ and $\epsilon_{j+1} \leq \epsilon_{j} / 2$;

- $g_{j+1} \in B_{\rho}\left(g_{j}, \epsilon_{j}\right) \cap T_{j+1}$;

- $B_{\rho}\left(g_{j+1}, 3 \epsilon_{j+1}\right) \subset T_{j+1}$

- $g_{j+1}\left|\Omega=g_{0}\right| \Omega$.

The sequence $\left\{g_{j}\right\}_{j \geq 1}$ converges uniformly to a map $g \in C(Z, M)$. It follows from the construction that $g\left|\Omega=g_{0}\right| \Omega$ and $g$ is $\epsilon$-close to $g_{0}$. Moreover, $\rho\left(g(x), g_{j}(x)\right) \leq$ $\sum_{i=j}^{\infty} \epsilon_{i} \leq 2 \epsilon_{j}$ for any $j \geq 1$ and $x \in Z$. Therefore, $g \in T_{j}, j \geq 1$. Since, by [17, Proposition 2.1], $\mathcal{K}_{\pi}\left(\mathbb{I}^{k}\right)$ is the intersection of all $T_{j}, g$ is as required.

The next step is to prove that if $f: N \rightarrow L$ is a perfect $P L$-map between simplicial complexes with the $C W$-topology, then $\mathcal{K}_{f}(L)$ is dense in $C(N, M)$. Recall that $f$ is a $P L$-map if $f(\sigma)$ is contained in a simplex of $L$ and $f$ is linear on $\sigma$ for every simplex $\sigma$ of $N$. In general, $N$ and $L$ are not metrizable, but all of their compact subsets are metrizable.

Lemma 2.2. Let $N, L$ be simplicial complexes and $f: N \rightarrow L$ a perfect $P L$-map. Then $\mathcal{K}_{f}(L)$ is dense in $C(N, M)$.

Proof. Fix $g \in C(N, M)$ and $\alpha \in C(N,(0,1])$. Following the proof of [1, Lemma 11.3], we are going to find a map $h \in \mathcal{K}_{f}(L)$ which is $\alpha$-close to $g$. To this end, let $L^{(i)}, i \geq 0$, denote the $i$-dimensional skeleton of $L$. We put $L^{(-1)}=\emptyset$ and $h_{-1}=g$. Construct inductively a sequence $\left(h_{i}: N \rightarrow M\right)_{i \geq 0}$ of maps such that

$$
\text { - } h_{i}\left|f^{-1}\left(L^{(i-1)}\right)=h_{i-1}\right| f^{-1}\left(L^{(i-1)}\right) \text {; }
$$


- $h_{i}$ is $\frac{\alpha}{2^{i+2}}$-homotopic to $h_{i-1}$;

- $h_{i} \in \mathcal{K}_{f}\left(L^{(i)}\right)$ for every $i$.

Assuming that the map $h_{i-1}: N \rightarrow M$ has been constructed, consider the complement $L^{(i)} \backslash L^{(i-1)}=\bigsqcup_{j \in J_{i}} \stackrel{\circ}{\sigma}_{j}$, which is the discrete union of open $i$-dimensional simplexes. Since $h_{i-1} \in \mathcal{K}_{f}\left(L^{(i-1)}\right)$, we can apply Lemma 2.1 for every simplex $\sigma_{j} \in L^{(i)}$ to find a map $g_{j}: f^{-1}\left(\sigma_{j}\right) \rightarrow M$ such that

- $g_{j}$ coincides with $h_{i-1}$ on the set $f^{-1}\left(\sigma_{j}^{(i-1)}\right)$;

- $g_{j}$ is $\frac{\alpha}{2^{i+2}}$-homotopic to $h_{i-1}$;

- all restrictions $g_{j} \mid f^{-1}(y), y \in \sigma_{j}$, are Krasinkiewicz maps.

Define a map $\varphi_{i}: f^{-1}\left(L^{(i)}\right) \rightarrow M$ by the formula

$$
\varphi_{i}(x)= \begin{cases}h_{i-1}(x) & \text { if } x \in f^{-1}\left(L^{(i-1)}\right) \\ g_{j}(x) & \text { if } x \in f^{-1}\left(\sigma_{j}\right) .\end{cases}
$$

It can be shown that $\varphi_{i}$ is $\frac{\alpha}{2^{i+2}}$-homotopic to $h_{i-1} \mid f^{-1}\left(L^{(i)}\right)$. By the Homotopy Extension Theorem, there exists a continuous extension $h_{i}: N \rightarrow M$ of the map $\varphi_{i}$ which is $\frac{\alpha}{2^{i+2}}$-homotopic to $h_{i-1}$. The map $h_{i}$ satisfies the inductive conditions.

Then the limit map $h=\lim _{i \rightarrow \infty} h_{i}: N \rightarrow M$ is well-defined, continuous and $\alpha$ close to $g$. Finally, since $h\left|f^{-1}\left(L^{(i)}\right)=h_{i}\right| f^{-1}\left(L^{(i)}\right)$ for every $i \geq 0, h \in \mathcal{K}_{f}(L)$.

Now we can complete the proof of Theorem 1.1. We use some arguments from the proof of [2, Proposition 3.4].

Proposition 2.3. Suppose $f: X \rightarrow Y$ is a perfect surjection between metric spaces. Then $\mathcal{K}_{f}(Y)$ is a dense and $G_{\delta}$-subset of $C(X, M)$.

Proof. Since $C(X, M)$ has the Baire property and $\mathcal{K}_{f}(Y)$ is the intersection of the open sets $\mathcal{K}_{f}(m, n, Y) \subset C(X, M), m, n \geq 1$, it suffices to show that each $\mathcal{K}_{f}(m, n, Y)$ is dense in $C(X, M)$. Let $\epsilon \in C(X,(0,1])$ and a $g \in C(X, M)$. We are going to find a map $h \in \mathcal{K}_{f}(m, n, Y)$ such that $\rho(g(x), h(x))<\epsilon(x)$ for all $x \in X$. Since $M$ is an $A N R, g$ can be approximated by simplicially factorizable maps (i.e., maps $g^{\prime} \in C(X, M)$ such that $g^{\prime}=g_{2} \circ g_{1}$, where $g_{1}$ is a map from $X$ into a simplicial complex $L^{\prime}$ and $\left.g_{2}: L^{\prime} \rightarrow M\right)$. So, $g$ itself can be assumed to be simplicially factorizable. Choose a simplicial complex $D$ and maps $g_{D}: X \rightarrow D$, $g^{D}: D \rightarrow M$ with $g=g^{D} \circ g_{D}$. The metric $\rho$ induces a continuous pseudometric $\rho_{D}$ on $D, \rho_{D}(x, y)=\rho\left(g^{D}(x), g^{D}(y)\right)$. Since $D$ is a neighborhood retract of a locally convex space, we can apply [1, Lemma 8.1] to find an open cover $\mathcal{U}$ of $X$ satisfying the following condition: if $\alpha: X \rightarrow K$ is a $\mathcal{U}$-map into a paracompact space $K$ (i.e., $\alpha^{-1}(\omega)$ refines $\mathcal{U}$ for some open cover $\omega$ of $\left.K\right)$, then there exists a map $q^{\prime}: G \rightarrow D$, where $G$ is an open neighborhood of $\overline{\alpha(X)}$ in $K$, such that $g_{D}$ and $q^{\prime} \circ \alpha$ are $\epsilon / 2$ close with respect to the pseudometric $\rho_{D}$. Let $\mathcal{U}_{1}$ be an open cover of $X$ refining $\mathcal{U}$ with $\operatorname{mesh} \mathcal{U}_{1}<1 / m$ and $\inf \{\epsilon(x): x \in U\}>0$ for all $U \in \mathcal{U}_{1}$.

Next, according to [1, Theorem 6], there exists an open cover $\mathcal{V}$ of $Y$ such that: for any $\mathcal{V}$-map $\beta: Y \rightarrow L$ into a simplicial complex $L$ we can find a $\mathcal{U}_{1}$-map $\alpha: X \rightarrow K$ into a simplicial complex $K$ and a perfect $P L$-map $p: K \rightarrow L$ with $\beta \circ f=p \circ \alpha$. We can assume that $\mathcal{V}$ is locally finite. Take $L$ to be the nerve of the cover $\mathcal{V}$ and $\beta: Y \rightarrow L$ the corresponding natural map. Then there exist a 
simplicial complex $K$ and maps $p$ and $\alpha$ satisfying the above conditions. Hence, the following diagram is commutative:

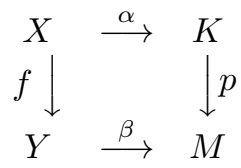

Since $K$ is paracompact, the choice of the cover $\mathcal{U}$ guarantees the existence of a map $\varphi_{D}: G \rightarrow D$, where $G \subset K$ is an open neighborhood of $\overline{\alpha(X)}$, such that $g_{D}$ and $h_{D}=\varphi_{D} \circ \alpha$ are $\epsilon / 2$-close with respect to $\rho_{D}$. Then, according to the definition of $\rho_{D}, h^{\prime}=g^{D} \circ \varphi_{D} \circ \alpha$ is $\epsilon / 2$-close to $g$ with respect to $\rho$. Replacing the triangulation of $K$ by a suitable subdivision, we may additionally assume that no simplex of $K$ meets both $\overline{\alpha(X)}$ and $K \backslash G$. So, the union $N$ of all simplexes $\sigma \in K$ with $\sigma \cap \overline{\alpha(X)} \neq \emptyset$ is a subcomplex of $K$ and $N \subset G$. Moreover, since $N$ is closed in $K, p_{N}=p \mid N: N \rightarrow L$ is a perfect map. Therefore, we have the following commutative diagram, where $\varphi=g^{D} \circ \varphi_{D}$ :

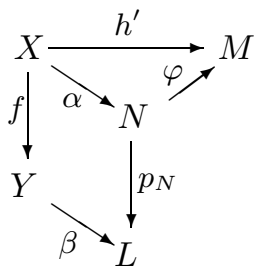

Using that $\alpha$ is a $\mathcal{U}_{1}$-map and $\inf \{\epsilon(x): x \in U\}>0$ for all $U \in \mathcal{U}_{1}$, we can construct a continuous function $\epsilon_{1}: N \rightarrow(0,1]$ with $\epsilon_{1} \circ \alpha \leq \epsilon$. By Lemma 2.2. there exists a map $\varphi_{1} \in C(N, M)$ which is $\epsilon_{1} / 2$-close to $\varphi$ and $\varphi_{1} \in \mathcal{K}_{p_{N}}(L)$. Let $h=\varphi_{1} \circ \alpha$. Then $h$ and $\varphi \circ \alpha$ are $\epsilon / 2$-close because $\epsilon_{1} \circ \alpha \leq \epsilon$. On the other hand, $\varphi \circ \alpha=h^{\prime}$ is $\epsilon / 2$-close to $g$. Hence, $g$ and $h$ are $\epsilon$-close.

It remains to show that $h \in \mathcal{K}_{f}(m, n, Y)$. To this end, fix $y \in Y$ and a continuum $P \subset f^{-1}(y)$ with $\operatorname{diam} h(P) \geq 1 / n$. Then $P^{\prime}=\alpha(P)$ is a continuum in $p_{N}^{-1}(\beta(y))$ which is not contained in any fiber of the restriction map $\varphi_{1} \mid p_{N}^{-1}(\beta(y))$. Since the last map is Krasinkiewicz, there exists a point $z^{*} \in P^{\prime}$ such that $C^{\prime} \subset P^{\prime}$, where $C^{\prime}$ is the component of $p_{N}^{-1}(\beta(y)) \cap \varphi_{1}^{-1}\left(\varphi_{1}\left(z^{*}\right)\right)$ containing $z^{*}$. Choose $x^{*} \in P$ with $\alpha\left(x^{*}\right)=z^{*}$ and a component $C$ of $f^{-1}(y) \cap h^{-1}\left(h\left(x^{*}\right)\right)$ which contains $x^{*}$. Then $\alpha(C)$ is a connected subset of $p_{N}^{-1}(\beta(y)) \cap \varphi_{1}^{-1}\left(\varphi_{1}\left(z^{*}\right)\right)$ meeting $C^{\prime}$. Consequently, $\alpha(C) \subset C^{\prime} \subset \alpha(P)$. Hence, $\alpha^{-1}(\alpha(x)) \cap P \neq \emptyset$ for any $x \in C$. Finally, since mesh $\mathcal{U}_{1}<1 / m$ and $\alpha$ is a $\mathcal{U}_{1}$-map, $\operatorname{diam} \alpha^{-1}(\alpha(x))<1 / m$ for all $x \in C$. So, $C \subset B(P, 1 / m)$. Therefore, $h \in \mathcal{K}_{f}(m, n, y)$, which completes the proof.

\section{PARAmetric Bing MaPs}

In this section we establish the proof of Theorem 1.2. Everywhere in the section, $(M, \rho)$ is a fixed complete $A N R$ free space, thus having the extension property from Section 2. Suppose $f: X \rightarrow Y$ is a perfect surjection and $d$ is a metric on $X$ generating its topology. For any $y \in Y$ and $m \geq 1$ let $\mathcal{B}_{f}(m, y)$ be the set of all maps $g \in C(X, M)$ such that:

- If $z \in M$ and $K_{0}, K_{1}$ are two continua in $f^{-1}(y) \cap g^{-1}(z)$ such that $K_{0} \cap$ $K_{1} \neq \emptyset$, then either $K_{0} \subset B\left(K_{1}, 1 / m\right)$ or $K_{1} \subset B\left(K_{0}, 1 / m\right)$. 
For any closed set $H \subset Y$ let $\mathcal{B}_{f}(m, H)=\bigcap_{y \in H} \mathcal{B}_{f}(m, y)$ and $\mathcal{B}_{f}(H)$ be the set of all $g \in C(X, M)$ such that $g \mid f^{-1}(y)$ is a Bing map for any $y \in H$.

Lemma 3.1. $\mathcal{B}_{f}(H)=\bigcap_{m \geq 1} \mathcal{B}_{f}(m, H)$.

Proof. It is easily seen that $\mathcal{B}_{f}(H) \subset \bigcap_{m \geq 1} \mathcal{B}_{f}(m, H)$. Assume $g \in \bigcap_{m \geq 1} \mathcal{B}_{f}(m, H)$ and $y \in H$. Then $g \mid f^{-1}(y)$ is a Bing map. Indeed, otherwise there exists $z \in M$ and two nontrivial continua $K_{0}, K_{1} \subset f^{-1}(y) \cap g^{-1}(z)$ with a common point such that $K_{i} \backslash K_{1-i} \neq \emptyset$ for $i=0,1$. Let $x_{i} \in K_{i} \backslash K_{1-i}$ and choose $m$ so big that $x_{i} \notin B\left(K_{1-i}, 1 / m\right), i=0,1$. So, $K_{i} \not \subset B\left(K_{1-i}, 1 / m\right)$ for any $i=0,1$, which contradicts the fact that $g \in \mathcal{B}_{f}(m, y)$.

Lemma 3.2. Each $\mathcal{B}_{f}(m, H)$ is open in $C(X, M)$.

Proof. We first need the following claim:

Claim 2. Let $g \in \mathcal{B}_{f}(m, y)$ for some $y \in Y$ and $m \geq 1$. Then there exists a neighborhood $V_{y}$ of $y$ in $Y$ and $\delta_{y}>0$ such that $y^{\prime} \in V_{y}$ and $g^{\prime} \in C(X, M)$ with $\rho\left(g^{\prime}(x), g(x)\right)<\delta_{y}$ for all $x \in f^{-1}\left(y^{\prime}\right)$ yields $g^{\prime} \in \mathcal{B}_{f}\left(m, y^{\prime}\right)$.

Indeed, otherwise we can find a local base $\left\{V_{k}\right\}_{k \in \mathbb{N}}$ of neighborhoods of $y$ in $Y$, points $y_{k} \in V_{k}$ and maps $g_{k} \in C(X, M)$ such that $\rho\left(g_{k}(x), g(x)\right)<1 / k$ for all $x \in f^{-1}\left(y_{k}\right)$ but $g_{k} \notin \mathcal{B}_{f}\left(m, y_{k}\right)$. Consequently, for any $k$ there exist $z_{k} \in M$, two continua $P_{k}^{0}, P_{k}^{1} \subset f^{-1}\left(y_{k}\right) \cap g_{k}^{-1}\left(z_{k}\right)$ having a common point, and points $x_{k}^{i} \in P_{k}^{i}$ with $d\left(x_{k}^{i}, P_{k}^{1-i}\right) \geq 1 / m, i=0,1$. Since the set $f^{-1}\left(\left\{y_{k}\right\}_{k \in \mathbb{N}} \cup\{y\}\right)$ is compact and contains all $P_{k}^{i}$, we may assume that each of the sequences $\left\{P_{k}^{i}\right\}$ and $\left\{x_{k}^{i}\right\}, i=0,1$, converges, respectively, to a continuum $P^{i} \subset f^{-1}(y)$ and a point $x^{i} \in P^{i}$. This implies that $\left\{z_{k}\right\}$ converges to a point $z \in M$ with $P^{i} \subset f^{-1}(y) \cap g^{-1}(z)$. Moreover, we have $P^{0} \cap P^{1} \neq \emptyset$ and $d\left(x^{i}, P^{1-i}\right) \geq 1 / m$ for any $i=0,1$. The last conditions contradict the fact that $g \in \mathcal{B}_{f}(m, y)$. This completes the proof of the claim.

Now, following the arguments from the proof of [21, Proposition 2.3] and using Claim 2 instead of [21, Lemma 2.2], we can establish that for any closed $H \subset Y$ the set $\mathcal{B}_{f}(m, H)$ is open in $C(X, M)$.

The proofs of the next two lemmas are similar to the proofs of Lemma 2.1 and Lemma 2.2 , respectively.

Lemma 3.3. Let $\pi: Z \rightarrow \mathbb{I}^{k}$, where $Z$ is a metric compactum and $k \geq 1$. Suppose $g_{0} \in C(Z, M)$ with $g_{0} \in \mathcal{B}_{\pi}\left(\mathbb{S}^{k-1}\right)$. Then for every $\epsilon>0$ there exists a map $g \in C(Z, M)$ such that $g \in \mathcal{B}_{\pi}\left(\mathbb{I}^{k}\right), g$ is $\epsilon$-homotopic to $g_{0}$ and $g \mid \pi^{-1}\left(\mathbb{S}^{k-1}\right)=$ $g_{0} \mid \pi^{-1}\left(\mathbb{S}^{k-1}\right)$.

Lemma 3.4. Let $N, L$ be simplicial complexes and $f: N \rightarrow L$ a perfect $P L$-map. Then $\mathcal{B}_{f}(L)$ is dense in $C(N, M)$.

Now we can complete the proof of Theorem 1.2.

Proposition 3.5. Suppose $f: X \rightarrow Y$ is a perfect surjection between metric spaces. Then $\mathcal{B}_{f}(Y)$ is a dense and $G_{\delta}$-subset of $C(X, M)$.

Proof. Since $\mathcal{B}_{f}(Y)$ is the intersection of the open sets $\mathcal{B}_{f}(m, Y), m \geq 1$, it suffices to show that each $\mathcal{B}_{f}(m, Y)$ is dense in $C(X, M)$. To this end, we repeat the construction and the notation from the proof of Proposition 2.3. Using Lemma 3.4 instead of Lemma 2.2, we obtain a map $\varphi_{1} \in C(N, M)$ such that $\varphi_{1} \in \mathcal{B}_{p_{N}}(L)$ and $\varphi_{1}$ is $\epsilon / 2$-close to $\varphi$. Let us show that the map $h=\varphi_{1} \circ \alpha$ belongs to $\mathcal{B}_{f}(m, Y)$. 
We fix $y \in Y$ and take two continua $P^{0}, P^{1} \subset f^{-1}(y) \cap h^{-1}(z)$ for some $z \in M$ such that $P^{0} \cap P^{1} \neq \emptyset$. Then $C^{0}=\alpha\left(P^{0}\right)$ and $C^{1}=\alpha\left(P^{1}\right)$ are nonempty continua in $p_{N}^{-1}(\beta(y)) \cap \varphi_{1}^{-1}(z)$ having a common point. Since $p_{N}^{-1}(\beta(y)) \cap \varphi_{1}^{-1}(z)$ is a Bing space, one of these continua is contained in the other one. Assume that $C^{0} \subset C^{1}$. Then $\alpha^{-1}(\alpha(x)) \cap P^{1} \neq \emptyset$ for all $x \in P^{0}$. Because all fibers of $\alpha$ are of diameter $<1 / m$ (recall that mesh $\mathcal{U}_{1}<1 / m$ ), we finally obtain that $P^{0} \subset B\left(P^{1}, 1 / m\right)$. Hence, $h \in \mathcal{B}_{f}(m, y)$, which completes the proof.

\section{Some mapping theorems For EXtensional Dimension}

In this section we apply Theorem 1.2 to extend some results from [14] which were established for maps between compact metric spaces. Extension theory, which was first introduced by Dranishnikov [5], is based on the following notion. If $K$ is a $C W$-complex we say that the extensional dimension of a space $X$ does not exceed $K$ (briefly, e- $\operatorname{dim} X \leq K$ ) provided $K$ is an absolute extensor for $X$. For example, $\operatorname{dim} X \leq n$ if and only if e-dim $X \leq S^{n}$. For a map $f: X \rightarrow Y$ we write e-dimf $f \leq K$ provided e-dim $f^{-1}(y) \leq K$ for all $y \in Y$.

We start with the following proposition established in [12, Theorem 1.2] for maps between compact metric spaces.

Proposition 4.1. Let $K$ be a $C W$-complex and $f: X \rightarrow Y$ be a perfect surjection between metric spaces with e-dim $f \leq K$. Then there exists an $F_{\sigma}$-set $A \subset X$ such that $\mathrm{e}-\operatorname{dim} A \leq K$ and $\operatorname{dim} f^{-1}(y) \backslash A \leq 0$ for all $y \in Y$.

Proof. According to [18], there exists a map $g: X \rightarrow Q$, where $Q=\mathbb{I}^{\omega}$ is the Hilbert cube, such that $g$ embeds every fiber $f^{-1}(y), y \in Y$. Let $g=\triangle_{i=1}^{\infty} g_{i}$ and $h_{i}=f \triangle g_{i}: X \rightarrow Y \times \mathbb{I}, i \geq 1$. Moreover, we choose countably many closed intervals $\mathbb{I}_{j}$ such that every open subset of $\mathbb{I}$ contains some $\mathbb{I}_{j}$. By [20, Lemma 4.1], for every $j$ there exists a 0 -dimensional $F_{\sigma}$-set $C_{j} \subset Y \times \mathbb{I}_{j}$ such that $C_{j} \cap\left(\{y\} \times \mathbb{I}_{j}\right) \neq \emptyset$ for every $y \in Y$. Now, consider the sets $A_{i j}=h_{i}^{-1}\left(C_{j}\right)$ for all $i, j \geq 1$ and let $A$ be their union. Since e-dimf $\leq K$, e-dim $h_{i} \leq K$ for any $i$. Hence, according to 3 , Corollary 2.5], e-dim $A_{i j} \leq K$ for all $i, j$. This implies that e-dim $A \leq K$.

It remains to show that $\operatorname{dim} f^{-1}(y) \backslash A \leq 0$ for every $y \in Y$. Let $\operatorname{dim} f^{-1}\left(y_{0}\right) \backslash A>$ 0 for some $y_{0}$. Since $g \mid f^{-1}\left(y_{0}\right)$ is an embedding, there exists an integer $i$ such that $\operatorname{dim} g_{i}\left(f^{-1}\left(y_{0}\right) \backslash A\right)>0$. Then $g_{i}\left(f^{-1}\left(y_{0}\right) \backslash A\right)$ has a nonempty interior in $\mathbb{I}$. So, $g_{i}\left(f^{-1}\left(y_{0}\right) \backslash A\right)$ contains some $\mathbb{I}_{j}$. Choose $t_{0} \in \mathbb{I}_{j}$ with $c_{0}=\left(y_{0}, t_{0}\right) \in C_{j}$. Then there exists $x_{0} \in f^{-1}\left(y_{0}\right) \backslash A$ such that $g_{i}\left(x_{0}\right)=t_{0}$. On the other hand, $x_{0} \in h_{i}^{-1}\left(c_{0}\right) \subset A_{i j} \subset A$, a contradiction.

For a set $A \subset X$ we write $\operatorname{rdim}_{X} A \leq n$ provided $\operatorname{dim} H \leq n$ for every closed subset $H$ of $X$ which is contained in $A$. The next theorem is an analogue of Theorem 1.9 from [14].

Theorem 4.2. Let $K$ be a $C W$-complex and $f: X \rightarrow Y$ a perfect map between metric spaces. Let $\tilde{X}=X \times \mathbb{I}$ and define $\tilde{f}: \tilde{X} \rightarrow Y$ by $\tilde{f}(x, t)=f(x)$. Consider the following properties:

(1) e- $\operatorname{dim} f \leq K$.

(2) Almost every map $g: \tilde{X} \rightarrow \mathbb{I}$ is such that e-dim $\tilde{f} \triangle g \leq K$.

(3) There is an $F_{\sigma}$-subset $A$ of $\tilde{X}$ with e-dim $A \leq K$ and $\operatorname{rdim}_{\tilde{X}} \tilde{f}^{-1}(y) \backslash A \leq 0$ for every $y \in Y$. 
(4) There is a $G_{\delta}$-subset $B$ of $\tilde{X}$ such that $\mathrm{e}-\operatorname{dim} B \leq K$ and $\operatorname{dim} \tilde{f}^{-1}(y) \backslash B \leq 0$ for every $y \in Y$.

Then $(1) \Rightarrow(2) \Rightarrow(3)$. Moreover, if $K$ is countable, then $(1) \Rightarrow(2) \Rightarrow(3) \Rightarrow(4)$.

Proof. To prove the implication $(1) \Rightarrow(2)$, take a map $g: \tilde{X} \rightarrow \mathbb{I}$ such that all restrictions $g \mid \tilde{f}^{-1}(y), y \in Y$, are Bing maps. So, any fiber $(\tilde{f} \triangle g)^{-1}(y, t)=g^{-1}(t) \cap$ $\left(f^{-1}(y) \times \mathbb{I}\right), t \in \mathbb{I}, y \in Y$, is a Bing space. Thus, it does not contain a nondegenerate interval. This implies that the projection of $(\tilde{f} \triangle g)^{-1}(y, t)$ onto $f^{-1}(y)$ is a 0 dimensional map. Then, by [6, Theorem 1.2], e-dim $\tilde{f} \triangle g \leq \mathrm{e}-\operatorname{dim} f^{-1}(y) \leq K$. Since $\mathbb{I}$ is a free space [11, by Theorem 1.2 , almost every map $g \in C(\tilde{X}, \mathbb{I})$ is such that the fibers of $\tilde{f} \triangle g$ are Bing spaces. The implication $(1) \Rightarrow(2)$ is established.

For the implication (2) $\Rightarrow(3)$, we fix a map $g: \tilde{X} \rightarrow \mathbb{I}$ such that e- $\operatorname{dim} \tilde{f} \triangle g \leq K$ and consider the family of all subintervals $\mathbb{I}_{j}$ of $\mathbb{I}$ with rational end-points. As in the proof of Theorem 4.1, for every $j$ there exists a 0-dimensional $F_{\sigma}$-set $C_{j} \subset$ $Y \times \mathbb{I}_{j}$ such that $\left(\{y\} \times \mathbb{I}_{j}\right) \cap C_{j} \neq \emptyset$ for every $y \in Y$. Let $C=\bigcup_{j \geq 1} C_{j}$ and $A_{1}=(\tilde{f} \triangle g)^{-1}(C)$. Since $\operatorname{dim} C=0$, it follows from [3, Corollary 2.5] that e$\operatorname{dim} A_{1} \leq K$. On the other hand, by Theorem 4.1, there exists an $F_{\sigma}$-subset $A_{2} \subset \tilde{X}$ such that e- $\operatorname{dim} A_{2} \leq K$ and $\operatorname{dim}(\tilde{f} \triangle g)^{-1}(y, t) \backslash A_{2} \leq 0$ for all $(y, t) \in Y \times \mathbb{I}$. Then $A=A_{1} \cup A_{2}$ is an $F_{\sigma}$-set in $\tilde{X}$ and e- $\operatorname{dim} A \leq K$. It remains to show that $\operatorname{rdim}_{\tilde{X}} \tilde{f}^{-1}(y) \backslash A \leq 0$ for every $y \in Y$. So, we fix $y_{0} \in Y$ and a closed set $H \subset \tilde{X}$ which is contained in $\tilde{f}^{-1}\left(y_{0}\right) \backslash A$. Suppose $\operatorname{dim} H>0$. Then $H$ contains a nontrivial component of connectedness $T$ (recall that $H$ is compact because so is $\tilde{f}^{-1}\left(y_{0}\right)$ ). Since $(\tilde{f} \triangle g)^{-1}\left(y_{0}, t\right) \backslash A$ is 0 -dimensional for all $t \in \mathbb{I}, g(T)$ is a nontrivial subinterval of $\mathbb{I}$. So, $g(T)$ contains some $\mathbb{I}_{j}$. Consequently, $T \cap(\tilde{f} \triangle g)^{-1}\left(C_{j}\right) \neq \emptyset$, which is a contradiction. Hence, $\operatorname{rdim}_{\tilde{X}} \tilde{f}^{-1}\left(y_{0}\right) \backslash A \leq 0$.

Finally, let us show the implication (3) $\Rightarrow(4)$ provided $K$ is countable. Suppose $A \subset \tilde{X}$ is an $F_{\sigma}$-set such that e- $\operatorname{dim} A \leq K$ and $\operatorname{rdim}_{\tilde{X}} \tilde{f}^{-1}(y) \backslash A \leq 0$ for every $y \in Y$. Since $K$ is countable, every metric space of extensional dimension $\leq K$ has a completion with the same dimension [13. This fact easily implies that $A$ can be enlarged to a $G_{\delta}$-set $B \subset \tilde{X}$ with e- $\operatorname{dim} B \leq K$. Then $\tilde{f}^{-1}(y) \backslash B$ is an $F_{\sigma}$-subset of $\tilde{X}$ which is contained in $\tilde{f}^{-1}(y) \backslash A, y \in Y$. Hence, $\operatorname{dim} \tilde{f}^{-1}(y) \backslash B \leq 0$ because $\operatorname{rdim}_{\tilde{X}} \tilde{f}^{-1}(y) \backslash A \leq 0$.

For a subset $B \subset X$ and a $C W$-complex $L$ we write e-rdim ${ }_{X} B \leq L$ provided e- $\operatorname{dim} F \leq L$ for any closed set $F$ in $X$ which is contained in $B$. Our next result extends Theorem 1.5 from 14 .

Theorem 4.3. Let $K, L$ be two $C W$-complexes and $f: X \rightarrow Y$ a perfect surjection between metric spaces such that $\mathrm{e}-\operatorname{dim} f \leq K$ and $\mathrm{e}-\operatorname{dim} Y \leq L$. Then there exists a decomposition $\tilde{X}=X \times \mathbb{I}=A \cup B$ of $\tilde{X}$ such that e-dim $A \leq K$ and e-rdim $\tilde{X}_{\tilde{X}} B \leq L$. If in addition, $K$ is countable, $B$ can be chosen so that $\mathrm{e}-\operatorname{dim} B \leq L$. In such a case, e- $\operatorname{dim} \tilde{X} \leq K * L$.

Proof. According to Theorem 4.2(3), there exists an $F_{\sigma}$-set $A \subset \tilde{X}$ with e-dim $A \leq$ $K$ and $\operatorname{rdim}_{\tilde{X}} \tilde{f}^{-1}(y) \cap B \leq 0$ for every $y \in Y$, where $B=\tilde{X} \backslash A$. Then, $f \mid F$ is a perfect 0-dimensional map for every closed set $F \subset \tilde{X}$ which is contained in $B$. Hence, by [3, Corollary 2.5], e- $\operatorname{dim} F \leq L$. So, e-rdim $\tilde{X} B \leq L$.

If $K$ is countable, we apply Theorem 4.2(4) to find a $G_{\delta}$-set $A \subset \tilde{X}$ such that e- $\operatorname{dim} A \leq K$ and $\tilde{f} \mid B$ being 0-dimensional with $B=\tilde{X} \backslash A$. Let $B=\bigcup_{i \geq 1} B_{i}$ with 
each $B_{i} \subset \tilde{X}$ closed. Then all restrictions $\tilde{f} \mid B_{i}, i \geq 1$, are 0 -dimensional perfect maps. So, again applying [3, Corollary 2.5] we obtain e- $\operatorname{dim} B_{i} \leq L, i \geq 1$. Hence, e- $\operatorname{dim} B \leq L$. Finally, according to [8, Theorem A], e-dim $\tilde{X} \leq K * L$.

Let us also mention the following corollary of Theorem 4.3.

Theorem 4.4. Let $K, L$ be connected $C W$-complexes and $f: X \rightarrow Y$ a perfect surjection between metric spaces such that $\mathrm{e}-\operatorname{dim} f \leq K$ and $\operatorname{edim} Y \leq L$. If $X$ is finite-dimensional and $K$ is countable, then $\operatorname{edim} X \leq K \wedge L$.

Proof. We can repeat the proof of Theorem 1.6 from [14]. The only difference is that we now apply Theorem 4.3 instead of [14, Theorem 1.5] and the application of the extensivity criterion of Dranishnikov 4 is replaced by similar results of Dydak [7] concerning general metric spaces.

\section{REFERENCES}

1. T. Banakh and V. Valov, General Position Properties in Fiberwise Geometric Topology, arXiv:math.GT/10012522.

2. T. Banakh and V. Valov, Approximation by light maps and parametric Lelek maps, Topol. Appl. 157 (2010), 2325-2431.

3. A. Chigogidze and V. Valov, Extension dimension and $C$-spaces, Bull. London Math. Soc. 34, 6 (2002), 708-716. MR1924198 (2003e:55001)

4. A. N. Dranishnikov, Extending mappings into $C W$-complexes, Mat. Sb. 182, 9 (1991), 1300-1310 (in Russian); translation in Math. USSR. Sb. 74, 1 (1993), 47-56. MR1133570 (93a:55002)

5. A. N. Dranishnikov, The Eilenberg-Borsuk theorem for mappings into an arbitrary complex, Mat. Sb. 185, 4 (1994), 81-90. MR.1272187(95j:54028)

6. A. N. Dranishnikov and V. V. Uspenskij, Light maps and extensional dimension, Topol. Appl. 80, 1-2 (1997), 91-99. MR 1469470 (98j:54060)

7. J. Dydak, Cohomological dimension and metrizable spaces, Trans. Amer. Math. Soc. 337, 1 (1993), 219-234. MR.1153013 (93g:55001)

8. J. Dydak, Cohomological dimension and metrizable spaces. II, Trans. Amer. Math. Soc. 348, 4 (1996), 1647-1661. MR:1333390 (96h:55001)

9. J. Krasinkiewicz, On mappings with hereditarily indecomposable fibres, Bull. Polish Acad. Sci. Math. 44 (1996), 147-156. MR1416420 (97g:54016)

10. J. Krasinkiewicz, On approximation of mappings into 1-manifolds, Bull. Polish Acad. Sci. Math. 44, 4 (1996), 431-440. MR1420956 (97k:54011)

11. M. Levin, Bing maps and finite-dimensional maps, Fund. Math. 151, 1 (1996), 47-52. MR.1405520 (97e:54031)

12. M. Levin, On extensional dimension of maps, Topol. Appl. 103 (2000), 33-35. MR.1746915 (2001k:54058)

13. M. Levin, On extensional dimension of metrizable spaces, preprint.

14. M. Levin and W. Lewis, Some mapping theorems for extensional dimension, Israel J. Math. 133 (2003), 61-76. MR.1968422 (2004d:54032)

15. E. Matsuhashi, Krasinkiewicz maps from compacta to polyhedra, Bull. Pol. Acad. Sci. Math. 54, 2 (2006), 137-146. MR2266144 (2007i:54036)

16. E. Matsuhashi, Parametric Krasinkiewicz maps, cones and polyhedra, preprint.

17. E. Matsuhashi and V. Valov, Krasinkiewicz spaces and parametric Krasinkiewicz maps, arXiv:math.GN/08024436.

18. B. Pasynkov, On geometry of continuous maps of countable functional weight, Fundam. Prikl. Matematika 4, 1 (1998), 155-164 (in Russian). MR 1786441(2001i:54039)

19. J. Song and E. Tymchatyn, Free spaces, Fund. Math. 163 (2000), 229-239. MR1758326 (2001g:54011) 
20. M. Tuncali and V. Valov, On dimensionally restricted maps, Fund. Math. 175, 1 (2002), 35-52. MR.1971237 (2004b:54061)

21. V. Valov, Parametric Bing and Krasinkiewicz maps, Topol. Appl. 155, 8 (2008), 916-922. MR 2406399 (2009e:54052)

Department of Computer Science and Mathematics, Nipissing University, 100 College Drive, P.O. Box 5002, North Bay, ON, P1B 8L7, CANada

E-mail address: veskov@nipissingu.ca 